Journal of Mathematics and Statistics 8 (2): 195-197, 2012

ISSN 1549-3644

(C) 2012 Science Publications

\title{
Existence of a Total Order in Every Set
}

\author{
Abdelmadjid Boudaoud \\ Department of Mathematics, Faculty of Mathematics and Informatics, \\ Laboratory of Pure and Applied Mathematics (L.M.P.A.), \\ M'sila University, B.P. 166, Ichbilia, 28000 M'sila, Algeria
}

\begin{abstract}
Problem statement: The axiom of choice, guarantees that all set could be well-ordered, in particular linearly ordered. But the proof in this case was not effective, that was to say, non constructive. It was natural to ask if there was mathematics in which we could given a more constructive proof. Approach: We work in the Nelson's IST which was an extension of ZFC (Zermelo-Fraenkel set theory with the axiom of choice). In the theory of IST there were two primitive symbols st, $\in$ and the axioms of ZFC together with three axiom schemes which we call the Transfer principle $(\mathrm{T})$, the principle of Idealization $(\mathrm{I})$ and the principle of Standardization $(\mathrm{S})$. Results: In the framework of IST we could construct, without the use of the choice axiom, a total order on every set. Conclusion: The Internal Set Theory provides a positive answer to our question.
\end{abstract}

Keywords: Total order, axiom of choice, IST theory, axiom of standardization

\section{INTRODUCTION}

The axiom of choice (Fraisse, 2000; Hrbacek and Jech, 1999) is an important and fundamental axiom in set theory sometimes called Zermelo's axiom of choice. It was formulated by Zermelo in 1904 and it differs from the other principles of set theory in that it is not effective, that is, a proof requiring the axiom of choice is nonconstructive.

This study is placed in the framework of IS T (Diener and Diener, 1995; Nelson, 1977, Nelson and Rogers, 1988; Berg, 1992; Vath, 2007). Moreover, to prove the result announced above in the abstract, the principal tool is the use, instead of an infinite set $\mathrm{X}$, of a finite subset $\mathrm{F}=\left\{\mathrm{x}_{1}, \mathrm{x}_{2}, \ldots, \mathrm{x}_{\omega}\right\} \subset \mathrm{X}$ containing all standard elements of $X$, transfer principle and standardization principle.

\section{MATERIALS AND METHODS}

In the proof of the result announced in the abstract, we use the following tools:

- Theorem of Nelson (1977) that affirms: For all set $\mathrm{X}$ there is a finite set $\mathrm{F} \subset \mathrm{X}$ such that for all standard $\mathrm{x} \in \mathrm{X}$ we have $\mathrm{X} \in \mathrm{F}$. This theorem is one of the most interesting consequences of the Idealization principle (I)
- Standardization principle (S) which one uses, generally, to create a new mathematical objects, that is to say, it is a principle of construction

- Transfer principle (T) who has been used several times

\section{RESULTS}

Theorem: On every non empty set $\mathrm{X}$ we can construct a total order R. In particular, this total order can be chosen in such a way that $\mathrm{X}$ has a least element or at least and a greatest element.

Proof: Let $\mathrm{X}$ be any non empty set. Suppose, by transfer, that $\mathrm{X}$ is standard. If $\mathrm{X}$ is finite, then we can easily prove the theorem. Indeed, if $X=\left\{\alpha_{1}, \alpha_{2}, \ldots, \alpha_{m}\right\}$ is a standard finite set, then every element of $\mathrm{X}$ is standard and $\mathrm{m}$ is standard (Diener and Diener, 1995; Nelson, 1977) and we can, for instance, define on $X$ the following relation $\alpha_{1} \prec \alpha_{2} \prec \ldots \prec \alpha_{\mathrm{m}}$ whose graph is $\left\{\left(\alpha_{i}, \alpha_{j}\right)_{m \geq i \geq 1, m \geq j \geq i} \mid \alpha_{i}, \alpha_{j} \in X\right\}$. Clearly, this relation is a total order. Moreover, the least element is $\alpha_{1}$ and the greatest element is $\alpha^{\mathrm{m}}$.

Suppose that $X$ is infinite. Let $F=\left\{x_{1}, x_{2}, \ldots, x_{n}\right\}$ be a finite subset of $\mathrm{X}$ containing all standard elements of X (Diener and Diener, 1995). Let $G=\left\{\left(x_{i}, x_{j}\right)_{N \geq i \geq 1, N \geq j \geq i} \mid x_{i}, x_{j} \in F\right\}$ be a subset of $X \times X$ and

Corresponding Author: Boudaoud, A., Department of Mathematics, Faculty of Mathematics and Informatics, Laboratory of Pure and Applied Mathematics (L.M.P.A.), M'sila University, B.P. 166, Ichbilia, 28000 M'sila, Algeria 
let $\mathrm{G}^{\mathrm{s}}$ be the standardization of $\mathrm{G}$. It is known that $\mathrm{G}^{\mathrm{s}}$ defines a relation $\mathrm{R}$ from $\mathrm{X}$ to $\mathrm{X}$ as follows:

$$
x \operatorname{Ry} \text { iff }(x, y) \in G^{s}
$$

We prove that $\mathrm{X}$ is totally ordered by $\mathrm{R}$. Indeed, the relation is standard because $G^{s}$ is also. On the other hand, by construction, $\forall^{\text {st }} \mathrm{x} \in \mathrm{X}[(\mathrm{x}, \mathrm{x}) \in \mathrm{G}]$ i.e. $\forall^{\text {st }} \mathrm{x} \in \mathrm{X}$ $\left[(x, x) \in G^{s}\right]$ which entails $\forall^{\text {st }} x \in X[x R x]$. Then by transfer $\forall x \in X[x R x]$. Hence $R$ is reflexive.

Let $x, y$ and $t$ be three standard elements of $X$, such that $x$ Ry and $y$ Rt. Then, $x, y$ and $t$ are elements of $F$ and $(\mathrm{x}, \mathrm{y}) \in \mathrm{G}^{\mathrm{s}}, \quad(\mathrm{y}, \mathrm{t}) \in \mathrm{G}^{\mathrm{s}} \quad$ i.e. $\quad(\mathrm{x}, \mathrm{y}) \in \mathrm{G}$ and $(\mathrm{y}, \mathrm{t}) \in \mathrm{G}$. This implies that in $F$ the index of $x$ is less than or equal to the index of $y$ which is less than or equal to the index of $t$. Hence, the index of $x$ is less than or equal to the index of $\mathrm{t}$, which shows that $(\mathrm{x}, \mathrm{t}) \in \mathrm{G}$ and therefore ( $\mathrm{x}$, t) $\in G^{\text {s }}$ i.e. $x$ Rt. So we proved:

$$
\forall^{\text {st }}(\mathrm{x}, \mathrm{y}, \mathrm{t}) \in \mathrm{X}^{3}[(\mathrm{xRy} \text { and } \mathrm{yRt}) \Rightarrow \mathrm{xRt}]
$$

Then by transfer:

$$
\forall(\mathrm{x}, \mathrm{y}, \mathrm{t}) \in \mathrm{X}^{3}[(\mathrm{xRy} \text { and } \mathrm{yRt}) \Rightarrow \mathrm{xRt}]
$$

Which implies that $\mathrm{R}$ is transitive.

Let $\mathrm{x}, \mathrm{y}$ be standard elements of $\mathrm{X}$ such that $\mathrm{xRy}$ and $y R x$. Then, $x, y$ is elements of $F$ and belongs to $G^{s}$ i.e. $(\mathrm{x}, \mathrm{y}),(\mathrm{y}, \mathrm{x})$ belong to $\mathrm{G}$.

This implies that in $F$ the index of $x$ is less than or equal to the index of $y$ and inversely. Hence, the index of $x$ equal to the index of $y$, which shows that $\mathrm{x}=\mathrm{y}$ and consequently $\mathrm{R}$ is anti-symmetric. Thus $\mathrm{R}$ is a partial order.

Prove that $\mathrm{R}$ is a total order on $\mathrm{X}$. Let $(\mathrm{y}, \mathrm{z})$ be a standard element of $\mathrm{X}^{2}$, then, $\mathrm{y}, \mathrm{z}$ are standard elements of $F$. Hence, $(y, z) \in G$ or $(z, y) \in G$ because in $F$ we have one and only one of the two following cases: the index of $\mathrm{y}$ is less than or equal to the index of $\mathrm{z}$ or inversely. This signifies that $\forall^{\text {st }}(y, z) \in X^{2}$ $\left[(y, z) \in G^{s}\right.$ or $\left.(z, y) \in G^{s}\right]$ which, by transfer, entails $\forall(y, z) \in X^{2} \quad\left[(y, z) \in G^{s}\right.$ or $\left.(z, y) \in G^{s}\right]$ i.e. $\forall(y, z) \in X^{2}$ [yRz or zRy] . Therefore, $\mathrm{R}$ is a total order on $\mathrm{X}$.

So that $\mathrm{R}$ has a least element, we choose $\mathrm{x}_{1}$ standard because in this case we have $\forall^{\text {st }} x \in X\left[x_{1} R x\right]$. Then by transfer $\forall \mathrm{x} \in \mathrm{X}\left[\mathrm{x}_{1} \mathrm{Rx}\right]$ which shows that $\mathrm{x}_{1}$ is a least element for $\mathrm{X}$. Analogously, so that $\mathrm{R}$ has a least element and a greatest element we choose $x_{1}$ and $x_{N}$ two standard elements. By transfer, we conclude for all $\mathrm{X}$.
Remark: Recall that by induction it is proven that "Every finite system of sets has a choice function" (Hrbacek and Jech, 1999). Therefore, when the system of sets is finite the existence of the choice function is provable and does not need to be deducted by an axiom. Let us show in the following how, in the previous proof, we choose $\mathrm{x}_{1}$ standard then $\mathrm{x}_{1}$ and $\mathrm{x}_{\mathrm{N}}$ standard.

$\mathrm{x}_{1}$ standard. Consider in this case the finite system of sets $\{X\}$ which is standard. Then there exists a choice function $\mathrm{f}_{1}$ which is by transfer standard such that $\mathrm{f}_{1}(\mathrm{X}) \in \mathrm{X}$. Put $\mathrm{x}_{1}=\mathrm{f}_{1}(\mathrm{X})$. Then $\mathrm{x}_{1}$ is by transfer standard. Let us take $\tilde{\mathrm{F}}=\left\{\mathrm{y}_{1}, \mathrm{y}_{2}, \ldots, \mathrm{y}_{\mathrm{m}}\right\}$ a finite subset of $\mathrm{X} \backslash\left\{\mathrm{x}_{1}\right\} \quad$ containing all standard elements of $\mathrm{X} \backslash\left\{\mathrm{x}_{1}\right\}$. Now, we can take the finite set $\mathrm{F}$ given above equal to $\left\{\mathrm{x}_{1}, \mathrm{y}_{1}, \mathrm{y}_{2}, \ldots, \mathrm{y}_{\mathrm{m}}\right\}$. Thus, $\mathrm{F}$ is a finite set such that the element having the smallest index (i.e. The index equals to 1) is standard.

$\mathrm{x}_{1}$ and $\mathrm{x}_{\mathrm{N}}$ are standard. As in the case (a), for the system $\{X\}$ there exists a choice function $\mathrm{f}_{1}$ which is by transfer standard such that the element $x_{1}=f_{1}(X) \in X$ is by transfer standard. Now, since $\mathrm{X} \backslash\left\{\mathrm{x}_{1}\right\}$ is standard different from $\phi$ then for the system $\left\{X \backslash\left\{x_{1}\right\}\right\}$ there exists a choice function $\mathrm{f}_{2}$ which is by transfer standard such that the element $x_{N}=f_{2}\left(X \backslash\left\{x_{1}\right\}\right) \in X \backslash\left\{x_{1}\right\}$ is by transfer standard. Let us take $\tilde{\mathrm{F}}=\left\{\mathrm{y}_{1}, \mathrm{y}_{2}, \ldots, \mathrm{y}_{\mathrm{q}}\right\}$ a finite subset of $X \backslash\left\{x_{1}, x_{N}\right\}$ containing all standard elements of $X \backslash\left\{x_{1}, x_{N}\right\}$. Now, we can take the finite set $F$ given above equal to $\left\{\mathrm{x}_{1}, \mathrm{y}_{1}, \mathrm{y}_{2}, \ldots, \mathrm{y}_{\mathrm{q}}, \mathrm{x}_{\mathrm{N}}\right\}$. Thus, $\mathrm{F}$ is a finite set such that the element having the smallest index (i.e. the index equals to 1) is standard and the element having the greatest index is also standard.

\section{DISCUSSION}

The absence of the axiom of choice, in our abovestated theorem, called the three axioms of the IST. This shows the fundamental role that plays this axiom in the set theory. Moreover, the previous techniques are always valid to get other results.

\section{CONCLUSION}

The Internal Set Theory has been adopted by quite a number of working mathematicians and this number becomes more and more important. This because it appears to be an efficient tool in several problems of mathematics, where we notice that most of proofs are characterized by the fact that they are constructive. 
In our case the most decisive tool, next to the other principles of IST, is the replacement of an infinite set by one of its finite parts which contains all its standard elements. In addition of this, we also find that the standardization principle is of a particular importance because it gives birth to new objects.

\section{REFERENCES}

Berg, I.P.V.D., 1992. Extended use of IST. Ann. Pure Applied Logic., 58: 73-92. DOI: 10.1016/01680072(92)90035-X

Diener, F. and M. Diener, 1995. Nonstandard Analysis in Practice. 1st Edn., Springer, Berlin, ISBN-10: 0387602976, pp: 250.
Fraisse, R., 2000. Theory of Relations. 1st Edn., Elsevier, New York, ISBN: 9780444505422, pp: 451.

Hrbacek, K. and T.J. Jech, 1999. Introduction to set theory. 3rd Edn., Dekker, New York, ISBN-10: 0824779150, pp: 291.

Nelson, E., 1977. Internal set theory: A new approach to nonstandard analysis. Bull. Amer. Math. Soc., 83: 1165-1198.

Nelson, E. and H. Jr. Rogers, 1988. The syntax of nonstandard analysis. Annals Pure Applied Logic., 38: 123-134.

Vath, M., 2007. Nonstandard analysis. Birkhauser Verlag Basel. Boston, Berlin. 